\title{
1 Circadian rhythms and the kidney
}

2

3 Dmitri Firsov ${ }^{1}$ and Olivier Bonny ${ }^{1,2}$

4

5 1Department of Pharmacology and Toxicology, University of Lausanne, Lausanne,

6 Switzerland

7

8 2Service of Nephrology, Department of Medicine, Lausanne University Hospital, Lausanne,

9 Switzerland

10

11

e-mail: Dmitri.firsov@unil.ch; Olivier.bonny@unil.ch

12 


\section{Abstract}

2 Numerous physiological functions exhibit substantial circadian oscillations. In the

3 kidneys, renal plasma flow, the glomerular filtration rate as well as tubular reabsorption

4 and/or secretion processes have been shown to peak during the active phase and decline

5 during the inactive phase. These functional rhythms are driven, at least in part, by a self-

6 sustaining cellular mechanism termed the circadian clock. The circadian clock controls

7 different cellular functions, including transcription, translation and protein post-

8 translational modifications (phosphorylation, acetylation, ubiquitylation, and so on) and

9 degradation. Disruption of the circadian clock in animal models results in the loss of blood

10 pressure control and substantial changes in the circadian pattern of water and electrolyte

11 excretion in the urine. Kidney-specific suppression of the circadian clock in animals

12 implicates both the intrinsic renal and extra-renal circadian clocks in these pathologies.

13 Alterations in the circadian rhythm of renal functions are associated with the

14 development of hypertension, chronic kidney disease, renal fibrosis and kidney stones.

15 Furthermore, renal circadian clocks might interfere with the pharmacokinetics and/or

16 pharmacodynamics of various drugs and are therefore an important consideration in the

17 treatment of some renal diseases or disorders.

18

19

20

21 


\section{Introduction}

2 The primary function of the kidneys is to maintain the homeostasis of extracellular fluids.

3 The traditional concept of homeostasis is centered on the idea that water and solute

4 balance in the body is achieved through numerous self-adjusting renal mechanisms that

5 are based on negative feed-back control - that is, homeostatic reactions are activated in

6 response to a change that has already occurred. However, research indicates that 7 adaptation of the kidneys to dramatic homeostatic transitions that are induced by 8 circadian rhythms in activity and feeding rely, at least in part, on a self-sustaining 9 molecular mechanism that continuously adjusts both glomerular and tubular functions in 10 anticipation of these circadian changes. This molecular mechanism, termed the circadian 11 clock, has been found in most tissues, including the kidneys. Peripheral circadian clocks 12 are thought to coordinate peripheral physiological functions with the pattern of activity 13 and/or feeding, which is, in turn, synchronized with circadian oscillations in 14 environmental variables by the central circadian clock in the suprachiasmatic nucleus in 15 the brain (Figure 1). Here, we summarize current knowledge of renal circadian rhythms 16 and renal circadian clock mechanisms. We also review the implications of disrupting 17 circadian rhythms or the circadian clock mechanism for the development and progression of kidney diseases in humans.

\section{Circadian rhythms in the kidney}

\section{Biological rhythms}

24 Most, if not all, vital functions in animals and plants show significant periodic fluctuations.

25 These functional fluctuations are the result of evolutionary adaptation to periodic changes in environmental variables to which all life on Earth is exposed. Geophysical cycles are by far the most important source of these environmental oscillations, including the $\sim 24$ h light-dark cycle caused by the Earth's rotation on its axis, the seasonal changes imposed by the combined effect of the $\sim 365$ day orbit of the Earth around the Sun and the tilting of the Earth on its axis and the well-known but still poorly understood effects of the lunar cycle. Importantly, many of the functional rhythms are self-sustaining and continue 
to oscillate even in the absence of external environmental stimuli, which suggests that the ability to anticipate the geophysical rhythms probably offered a selective advantage.

All biological rhythms can be classified on the basis of their period length, that is, circadian rhythms $(\sim 24 \mathrm{~h})$, infradian rhythms $(>24 \mathrm{~h})$ and ultradian rhythms, $(<24 \mathrm{~h})$. In this Review, we focus on circadian rhythms in the kidneys, whereas other types of renal functional rhythms, such as infradian rhythms, are reviewed elsewhere (1).

\section{Functional rhythms in the kidney}

Functional circadian oscillations are probably most easily seen in the kidneys, as a marked reduction occurs in the volume of urine excreted during the night compared to during the day (Figure 2). The first scientific description of circadian rhythms in the kidney was published in the mid- $19^{\text {th }}$ century by Edward Smith, a British physician who detected urinary urea and water excretion rhythms by analyzing urine samples that were collected hourly from prisoners in London's Coldbath Fields prison (2). Since then, circadian oscillations have been documented for most renal functions.

We now know that multiple renal processes show circadian rhythms, including the glomerular filtration rate (GFR), renal plasma flow (RPF) and renal excretion of water and major urinary solutes (reviewed in (3-8)). Most renal functional rhythms have similar kinetics, reaching their peak value in the middle of the phase of maximal behavioural activity and reaching a trough during the phase of minimal behavioural activity, which are hereafter referred to as the active and inactive phases, respectively. Of note, the active phase in humans and other diurnal animals is during the day, whereas it is during the night in nocturnal animals, such as rats and mice. However, the amplitude of the oscillations in different renal functional variables differs substantially. For example, the amplitude of circadian oscillations in GFR and RPF is 50\% (9-11), whereas the rate of water and major electrolyte excretion is several-fold higher during the active phase than during the inactive phase (12-15). In mice, circadian oscillations in the inner medullary concentrations of sodium, chloride and urea exist, resulting in a substantial increase in the inner medullary osmolality during the active phase (16). These findings suggest that the cortico-medullary gradient in the kidneys parallels the oscillations in GFR, thereby facilitating water reabsorption when the GFR and, hence, the filtered load of water are substantially increased. Experiments using oxygen-sensitive carbon paste electrodes 
renal medulla follow the circadian pattern of the RPF (17). As the oscillations in renal oxygenation parallel those of nutrient delivery to the kidneys, these data strongly suggest that renal energy production also follows a circadian pattern. Importantly, these results might have clinical relevance, as they suggest that the kidney is more vulnerable to hypoxic stress during the night.

Thus, a growing body of evidence suggests that the homeostatic control of extracellular fluid volume and composition involves continuous adjustment of diverse renal functions throughout the $24 \mathrm{~h}$ circadian cycle. Current research in renal circadian physiology is focused on identifying the molecular mechanisms underlying circadian rhythmicity of renal functions, determining whether renal functional rhythms are driven by systemic circadian time cues (activity, food components, hormones, body temperature and so on) or by local mechanisms and whether disruption of circadian rhythms causes human kidney diseases.

Although it is almost impossible to isolate the kidneys from factors that might be involved in the generation and/or maintenance of circadian oscillations, it is clear that the GFR rhythm is independent of systemic blood pressure oscillations and of sympathetic nervous system activity $(18,19)$. Furthermore, the circadian rhythm of potassium excretion in the urine is not influenced by posture or feeding pattern (20). These studies suggest the existence of a self-sustaining molecular mechanism that is involved in the circadian control of renal functions. The discovery of a ubiquitous, highly conserved molecular core of this mechanism, which was termed the circadian clock, was a significant step forward in the field, which was recognized with the award of the 2017 Nobel Prize in Physiology and Medicine (21).

\section{Transcriptional oscillations in the kidneys}

The circadian clock is a network of interconnected transcription feedback loops that generate cell-autonomous, self-sustaining transcriptional circadian oscillations (reviewed in $(22,23)$ ). In mammals, the main feedback loop is activated by a heterodimeric transcription activator comprising brain and muscle ARNT-like 1 (BMAL1) and circadian locomoter output cycles protein kaput (CLOCK) (Figure 1). BMAL1-CLOCK

31 heterodimers trigger the transcription of a wide range of circadian clock-controlled genes (CCGs), including the period (PER1, PER2 and PER3) and cryptochrome (CRY1 and CRY2) 
1 activity of BMAL1-CLOCK, thereby forming the inhibitory limb of the feedback loop.

2 Another important feedback mechanism involves the nuclear receptors REV-ERB and ROR, which control the transcription of BMAL1. This core clock circadian machinery is ubiquitously expressed, including throughout the central nervous system and peripheral tissues, as well as in the kidneys (24-26). All individual cellular clocks are synchronized to each other and to geophysical time through the activity of a central oscillator termed the 'master clock', which is located in the suprachiasmatic nucleus (SCN) of the hypothalamus (reviewed in (27)). The master clock, in turn, is continuously synchronized to geophysical time by photic (light-dark) cues that are perceived by the retina and are transmitted to the SCN through the optic nerve (28). The major signals transmitted by the master clock to reset the peripheral oscillators include circadian synthesis and/or release of circulating factors (hormones, food components, food metabolites and so on), circadian control of neuronal activity (including control of activity and feeding behaviour) and circadian control of body temperature (reviewed in (29)). Collectively, the SCN and the extra-SCN circadian oscillators constitute a hierarchically organized circadian timing system that enables the coordination of most biological processes (at the cellular, tissue and organ levels) with geophysical time.

Current estimates of the number of CCGs vary substantially (from 10 to $100 \%$ of genes) depending on which algorithm is used to identify CCGs (reviewed in (30)). An analysis of the transcriptomes of 12 adult mouse organs showed that the transcription of $\sim 43 \%$ of all protein-coding genes in the mouse genome showed circadian oscillations in at least one of the organs tested (31). Importantly, only the liver exceeded the kidneys in the total number of circadian transcripts ( $\sim 16 \%$ in the liver versus $\sim 13 \%$ in the kidney), suggesting that robust circadian clock activity exists in renal cells. This and other studies $(32,33)$ also showed that the circadian rhythmicity was organ-specific for most oscillating transcripts, supporting the idea that the main role of the circadian clock is to make temporal adjustments to cell-type-specific and tissue-specific functions. Marked circadian oscillations in hundreds of mRNAs were identified in microdissected mouse renal tubules $(34,35)$, including in the distal convoluted tubule (DCT), the connecting tubule (CNT) and the cortical collecting duct (CCD), and genetic inactivation of Clock leads to dramatic changes in the transcriptome of these tissues(34). These transcriptome-wide studies and other targeted, gene-specific analyses of transcripts encoding renal transporters or transport-related proteins have revealed rhythmic expression of renal 
1 vasopressin V1a receptor (Avpr1) and Avpr2(16, 34), urea transporter 2 (Ut2; also known

2 as Slc14a2) (16), potassium-transporting ATPase alpha chain 2 (Atp12a)(36), the aquaporins Aqp1, Aqp2 and Aqp3(10, 16, 34), epithelial sodium channel subunit alpha ( $\alpha$ ENAC; encoded by Scnn1a)(10) and ubiquitin carboxyl-terminal hydrolase 2 (Usp2)(37).

\section{Post-transcriptional circadian rhythms}

Circadian oscillation in transcription is not the only cellular mechanism that couples renal functions to circadian rhythms in environmental factors - translational and posttranslational regulations are also crucially important (Figure 3). For example, the circadian clock imposes rhythmic transcription and translation on RNAs that are involved in ribosome biogenesis in the mouse liver $(38,39)$. As the peak of ribosome biogenesis and polysome formation occurs in the middle of the active phase, the circadian clock likely coordinates the energy-consuming process of protein synthesis with the circadian pattern of energy production in cells. Ribosome profiling in mouse kidneys to identify rhythmically translated renal mRNAs found that $41 \%$ of circadian transcripts are rhythmically translated and 55\% of rhythmically translated transcripts had a circadian expression pattern(40). This analysis revealed rhythmic transcription and translation of several key genes or proteins that are involved in many renal homeostatic functions, including podocin(Nphs2), the aquaporins (Aqp2, Aqp4 and -Aqp8), claudin 1(Cldn1), serum glucocorticoid-regulated kinase 1 (Sgk1), mitochondrial 1,25-dihydroxyvitamin $\mathrm{D}_{3}$ 24-hydroxylase (Cyp24a1), the urate transporter GLUT9(Glut9; also known as Slc2a9) and many others. The category of rhythmically translated transcripts that do not cycle at the transcription level (and thus are not detected by RNA profiling as genes that are relevant for circadian physiology) is of special interest, and include the adenosine A1 receptor (Adora1), the prostaglandin E2 receptors EP3 (Ptger3) and EP4 (Ptger4), prostaglandin $\mathrm{F}_{2} \alpha$ receptor (Ptgfr), peroxisome proliferator receptor activator alpha (Ppara), claudin 19 (Cldn19), the $\mathrm{b}^{0,+}$ amino acid transporter (Slc7a9), the sodium-driven anion-exchanger NDCBE (Slc4A8), the peptide transporter PEPT1 (Pept1) and the TASK2 (Task2) potassium channel. Overall, this study showed that $\sim 1,000$ renal transcripts (of $\sim 12,000$ detected transcripts) are translated in a circadian pattern. However, these results must be interpreted with caution, as protein levels in a cell might be strongly influenced by 
other factors, such as protein degradation and/or protein secretion. Therefore, precise analysis of the circadian proteome of the kidney awaits further investigation.

The wide range of post-translational modifications that might contribute substantially to periodic changes in protein stability, subcellular localization, proteinprotein interactions and protein function, are another level of complexity to consider when correlating circadian translation or proteome data with functional circadian oscillations in renal functions. For example, targeted analysis of the WNK4-OSR1-SPAKsodium chloride co-transporter (NCC) signalling cascade, which controls sodium reabsorption in the distal convoluted tubule, showed circadian oscillations in the levels of phosphorylated OSR1, SPAK and the active form of NCC, but not in their total protein levels $(41,42)$.

Although substantial progress has been made in defining the role of posttranslational modifications in regulation of core clock proteins(reviewed in (43), little is known about circadian patterns of post-translational modifications in the entire proteome. Global analyses of the circadian phosphorylome and the circadian acetylome have been carried out in the mouse liver and identified $\sim 20,000$ phosphorylation sites (in $\sim 4,400$ liver proteins), $\sim 25 \%$ of which are regulated in a circadian manner(44). Of note, the amplitude of phosphorylation cycles substantially exceeded the amplitudes of the cycles in the circadian transcriptome and circadian proteome in the same tissue. Analysis of the liver acetylome detected circadian oscillations in $\sim 13 \%$ of $\sim 1,000$ acetylation sites in liver proteins (45). Analysis of the molecular pathways affected by circadian acetylation revealed an enrichment of proteins involved in the urea and tricarboxylic acid cycles and in the metabolism of amino acids and lipids. Collectively, these studies open new avenues of research in circadian physiology and provide technical solutions to study the role of circadian post-translational modifications in other tissues, including the kidney.

\section{[H2] Intrinsic versus external regulation}

As discussed above, circadian oscillations in the kidney can be entrained by intrinsic renal circadian clocks and/or by external circadian time cues. Circulating hormones are the most obvious candidates for the external circadian entrainment, as most hormones show substantial circadian fluctuations in their plasma levels. One of the best-known examples is aldosterone, a mineralo-corticosteroid hormone that is secreted by the adrenal glands 
1 blood pressure control. Aldosterone regulates apical sodium entry in principal cells of the

2 collecting duct and in the connecting tubule via the epithelial sodium channel ENaC

3 (reviewed in (46)). Plasma aldosterone levels peak in the first half of the active phase (47,

4 48), a pattern that parallels that of the GFR and, hence, of the filtered sodium load. Thus,

5 circadian oscillations in plasma aldosterone levels might be necessary for the continuous

6 adaptation of tubular sodium reabsorption capacity to circadian oscillations in the filtered sodium load. Plasma aldosterone levels are dramatically increased and circadian aldosterone oscillations are substantially reduced in mice deficient in CRY1 and CRY2 (Cry1/Cry2-null mice)(48). Analysis of the circadian transcriptome of the adrenal glands in these mice detected a chronic increase in the expression of the aldosterone biosynthetic enzyme type VI 3-hydroxyl-steroid dehydrogenase (HSD3B6). Functional defects in Cry1/Cry2-null mice included salt-sensitive hypertension and a non-dipping pattern of arterial blood pressure. This study was the first to show that an extra-renal circadian clock controlled a vital renal function through the circadian synthesis and release of a hormonal mediator. Another study in mice provided evidence that a circadian rhythm in oxygen levels in the circulation and in kidney tissue might entrain the intrinsic renal circadian clocks by inducing circadian oscillations in the levels of hypoxia-inducible factor $1 \alpha$ (HIF $1 \alpha)$ (49); the nuclear levels of HIF $1 \alpha$ in the kidney oscillated with a peak in the first half of the active phase. In addition, exposure of cultured cells to circadian oscillations in physiological oxygen resynchronized the individual cellular circadian clocks to follow the oxygen rhythm, an effect that was abolished by knockdown of HIF1 $\alpha(49)$. Interestingly, acid produced during hypoxia was recently shown to decrease the translation of clock constituents by suppressing mTORC1 signaling, inducing a disruption of the circadian clock (50)

Other studies have characterized the role of the core clock components PER1, CLOCK and BMAL1 in renal function. PER1 positively regulates aldosterone synthesis in an adrenal cell line and plasma aldosterone levels in vivo(51). Functional analysis of Per1null mice in conditions of high-salt and mineralocorticoid treatment revealed a nondipping hypertension resulting from PER1 deficiency(52) (53). Furthermore, PER1 controls the transcription of the genes encoding several key proteins involved in solute reabsorption along the nephron, including $\alpha \operatorname{ENAC(54-56),~NCC(57),~the~kinases~with~no~}$ lysine 1 (WNK1) and WNK4(57), sodium/hydrogen exchanger 3 (NHE3)(58), sodium- 
1 glucose cotransporter 1 (SGLT1)(58) and a group of genes that are involved in the endothelin axis of sodium reabsorption in the kidney(59).

A detailed renal phenotyping of Clock-null mice $(12,34)$ demonstrated that CLOCK deficiency results in the loss of circadian rhythmicity of urinary excretion of sodium, potassium and water. In addition, the circadian rhythm in plasma aldosterone levels was disrupted and blood pressure was substantially reduced in Clock-null mice. Transcriptome analysis of the kidneys of Clock-null mice revealed substantial changes in the expression levels and/or in the circadian expression patterns of Avpr1, Avpr2, Aqp2, Aqp4, Ppara, Slc9a3 (which encodes NHE3) and many other transcripts that are important for diverse renal functions. Pathway enrichment analysis identified a group of renal cytochrome p450 enzymes (Cyp4a12a, Cyp4a12b, Cyp4a14, Cyp2c44 and Cyp2j13) that are involved in the conversion of arachidonic acid to different active metabolites, including 20-hydroxyeicosatetraenoic acid (20-HETE). In the kidneys of Clock-null mice, the circadian rhythm of 20-HETE exhibited a substantial shift in acrophase and a substantial reduction in average $24 \mathrm{~h}$ levels. As 20-HETE is a potent modulator of glomerular filtration and tubular reabsorption (60-62), these results suggest that regulation of 20HETE synthesis is one of the crucial mechanisms by which the circadian clock controls renal function. Increased renal fibrosis and renal parenchymal damage in Clock-null mice after unilateral ureteral obstruction (a model of renal fibrosis) suggests that the circadian clock also has a role in renal fibrosis (63). At the molecular level, the circadian clock inhibits the transforming growth factor- $\beta$ (TGF $\beta$ )-cyclooxygenase 2 (COX2; also known as PTGS2) profibrotic axis in the kidney(63), leading these researchers to suggest that the renal circadian clock might be a therapeutic target for treatment of chronic kidney disease (CKD).

BMAL1 is thought to be the sole indispensable component of the core clock machinery (64). Bmal1-null mice have a multitude of mild-severe dysfunctions, including arrhythmic behaviour (64), impaired metabolism of glucose (65) and fatty acids (66), early aging $(67,68)$ and fertility problems (69). Consequently, the general health impairment in Bmal1-null mice complicates analysis of organ-specific phenotypes. Circadian rhythmicity in the medullary concentration of sodium and urea and in the corticomedullary osmotic gradient is lost in Bmal1-null mice(16), and molecular analyses revealed blunted circadian expression of Avpr1, Avpr2, Aqp2 and Slc14a2 in the kidneys of Bmal1-null mice. However, these data must be interpreted cautiously, as other factors 
1 in addition to renal medullary circadian clocks might be involved in the circadian rhythmicity of corticomedullary osmolality, including circadian oscillations in blood pressure, GFR and RPF. Interestingly, Bmal1-null mice exhibited a non-dipping blood pressure pattern and a substantial reduction in blood pressure (70), a result that was confirmed and extended in mice with a smooth muscle-specific knockout of Bmal1, which also had a similar blood pressure phenotype(71). However, a detailed analysis of renal function was not carried out in either of these Bmal1-null mice strains.

The role of the kidney in circadian oscillations of the main circulating components of the renin-angiotensin-aldosterone system (RAAS) and in blood pressure rhythms was examined in two mouse strains with kidney cell-specific knockout of Bmal1. To specifically disrupt the circadian clock in renin-secreting granular cells, mice that constitutively express Cre recombinase from the promoter of the renin $1^{\mathrm{d}}\left(\operatorname{Ren} 1^{d}\right)$ gene(72) and carry a conditional allele of the Bmal1 gene(73) (Bmal1 ${ }^{\text {lox } / \text { lox } / \text { Ren } 1^{d} \text { Cre }}$ mice) were generated (72)(72)(72)(72)(71)(10). In these mice, the circadian pattern of renin protein expression in kidney tissue was disrupted and plasma aldosterone levels were moderately reduced. Analysis of urine samples revealed a mild polyuria, moderate changes in the circadian rhythm of urinary sodium excretion and low urinary $\mathrm{pH}$. In addition, the GFR was substantially increased and blood pressure was markedly decreased, although the dipping pattern of blood pressure was maintained. These results suggest that circadian oscillations in arterial blood pressure are driven mostly by circadian clocks in extra-renal tissues (adrenal glands, smooth muscle cells and possibly other tissues) rather than by intrinsic renal circadian clocks. Of note, the phenotypic differences between different constitutive knockout strains might be masked by adaptive mechanisms that are activated during mouse embryonic and postembryonic development.

In a second study(74), the role of intrinsic circadian clocks in renal tubular cells was examined using mice with a doxycycline-inducible, nephron-specific knockout of Bmal1 (Bmal1 lox/lox/Pax8-rtTA/LC1 mice)(75). In these mice, aside from reduced kidney size, disruption of clock activity in the nephron did not result in any overt renal abnormalities. Circadian patterns of urinary sodium, potassium and water excretion did not different from those of control animals, thereby providing evidence for extrarenal control of renal excretory rhythms. However, increased plasma creatinine and urea levels despite a normal GFR indicated that tubular function was impaired, and kidney 
1 transcriptome analysis detected enrichment of genes involved in fatty acid and amino acid metabolism and in organic anion transport. As renal metabolism relies predominantly on fatty acid oxidation, these results suggest that the intrinsic tubular circadian clocks have a substantial role in the control of metabolic processes in renal tubular cells. In support of this hypothesis, the $\mathrm{NAD}^{+} / \mathrm{NADH}$ ratio, which is a marker of the oxidative phosphorylation/glycolysis ratio and/or mitochondrial function, was substantially reduced in Bmal1 lox/lox/Pax8-rtTA/LC1 mice. The increase in plasma creatinine levels in these mice might be explained by a marked reduction in their expression of Slc22a8 (also known as Oat3), which encodes an organic anion transporter that contributes substantially to the basolateral secretion of creatinine in the proximal tubule in mice(76). Systolic, but not diastolic, blood pressure was modestly reduced in Bmal1 lox/lox/Pax8rtTA/LC1 mice, whereas the blood pressure circadian rhythm was preserved.

In general, studies of these two mouse strains with kidney-specific disruption of the circadian clock showed that intrinsic renal circadian clocks are important but not crucial for kidney function (at least in the absence of additional factors, such as pathology, stress and so on), and that most renal functional oscillations are entrained by external circadian time-cues.

\section{Clinical relevance}

Although circadian rhythms in several renal functions have been described, their clinical importance is not yet clear. Assigning renal or systemic circadian rhythms have important clinical implications when (i) the molecular clock is genetically altered, (ii) the circadian clock is misaligned with the biological clock (such as when time is shifted rapidly, for example after a flight across longitudes) or (iii) when the balance between the efficacy and the adverse effects of a given pharmacological agent needs to be finely tuned (such as in chemotherapy). As a substantial number of drugs are eliminated in the kidney, the plasma concentration or the bioavailability of these drugs might depend on the circadian expression of renal transporters. For instance, inactivation of Bmal1 in mouse renal tubules markedly affects the efficacy of the diuretic furosemide owing to a strong decrease in the levels of OAT3, the transporter that secretes furosemide in the tubular lumen (74).

Genetic alterations of the molecular clock are present in some individuals with the rare familial advanced sleep phase syndrome that is linked to mutations in PER2 (77), 
CRY2 (78) and casein kinase 1 delta (CSNK1D) (79), and with familial delayed sleep phase syndrome that is caused by mutations in CRY1, with a surprisingly high frequency of variants in the population (80). However, to the best of our knowledge, no renal phenotypes have been described in patients affected by these rare syndromes. In genome-wide association studies (GWAS), common variants in the genes encoding the core clock proteins BMAL1, CLOCK and NR1D1 and the ROR, PER and CRY family proteins have been associated with mental illnesses, central nervous system degeneration, sleep disorders and metabolic diseases (reviewed in (81)). Of note, variants in BMAL1 have been associated with hypertension and/or diabetes, two major risk factors for chronic kidney disease $(82,83)$. However, none of these genes or loci have been directly associated with kidney diseases (source: GWAS catalog(84)). However, GWAS are limited because only some specific SNPs in the core clock genes were analysed, and GWAS may missother possible genomic variants or translational and post-translational processes that might trigger the development of renal diseases.

Rapid changes in time zone, such as during an overseas flight, or chronic disruption of circadian rhythms, such as for night shift workers, might create conflicts between the external time and the endogenous molecular time and result in insulin resistance and hypertension (85). This outcome indicates that misalignment of external time cues and endogenous core clocks can increase the cardiovascular risks, largely confirming epidemiological data showing that night shift workers are at increased risk of obesity, diabetes and cardiovascular diseases. However, whether the kidneys are also more prone to disease in these circumstances is unknown.

\section{The circadian system in renal diseases}

Fibrosis. Alterations of the core clock can lead to tissue fibrosis (Table 1). For example, a natural mutation in the gene encoding casein kinase 1 epsilon, an important regulator of the core clock, causes severe cardiac and renal fibrosis that results in renal insufficiency and premature death in hamsters (86). In addition, indirect evidence points to a role for the molecular clock in exacerbating or enhancing fibrogenic repair processes after a first 'hit'. For example, unilateral ureteral obstruction results in more TGF $\beta$-dependent renal fibrosis in Clock-deficient mice than in wild-type littermates(63). The increased fibrosis in these mice was attributed to elevated expression of cyclooxygenase 2 (Cox2) and was 
rescued by treatment with the COX2 inhibitor celecoxib (63). Thus, CLOCK might have a

2 protective effect in renal fibrosis by controlling Cox2 expression. By contrast, deoxycorticosterone acetate (DOCA)-salt treatment resulted in more renal inflammation and fibrosis in wild-type animals than in mice with the hypomorphic Clock $\Delta 19$ allele, suggesting that CLOCK exacerbates renal fibrosis in this hypertension model (87). The apparently contradictory observations about the role of the clock in renal fibrosis in these two mouse models might be due to a direct effect of salt on the molecular clock, but differences in the genetic background of the two mouse strains might also have a role. Indeed, high-salt treatment alters the expression of several components of the core clock, establishing a link between the renal expression of core clock genes and environmental cues. In rats fed a high-salt diet, the time of peak Bmal1 expression was delayed by several hours and the expression of Cry1 and Per2 was completely lost in the inner medulla of the kidney, but not in the renal cortex. This effect seems to be dependent on the endothelin system and, in particular, the type B endothelin receptor(88). The absence of detectable expression of some components of the core clock in the renal medulla in mice fed a highsalt diet might be the result of the special role that this part of the kidney has in controlling the corticomedullary osmotic gradient. Furthermore, this gradient has been shown to be under the strict control of the circadian system(16). Thus, alterations of this osmotic gradient by salt intake or other gradient modulators might contribute to changes in the expression of some core clock genes and might modulate fibrosis. Intriguingly, studies have shown that the nervous system might mediate the effects of circadian clock disruption on renal injury and fibrosis. For example, exposure of mice to blue light prior to ischaemia-reperfusion reduced renal injury by decreasing neutrophil recruitment to the injured tissue. This effect was mediated, at least in part, by a sympathetic ( $\beta 3$ adrenergic receptor-dependent) pathway, independently of melatonin or corticosterone levels (89). A role for the sympathetic nervous system in renal inflammation is further strengthened by the finding that renal denervation alone reduced cardiac fibrosis and rescued blood pressure dipping in a rat model of metabolic syndrome (90).

Kidney stones. One of the most prevalent diseases of the urinary tract and one that results in substantial morbidity, kidney stones form when urine becomes supersaturated with salts, resulting in the generation of microcrystals. If the crystals aggregate and attach 
1 to the urothelium, the stones grow and might eventually detach when they reach a critical

2 size, blocking the ureter and inducing renal colic. Supersaturation depends on the concentration of solutes in urine, which changes during the day and follows a diurnal rhythm (highest during the day and lowest at night) (91-96). The concentration of an ion in the urine depends on the rate of its excretion and on urinary volume. Furthermore, urinary $\mathrm{pH}$ also strongly affects lithogenic risk by modulating salt supersaturation, and also displays a circadian rhythm $(97,98)$. Overall, the risk of forming kidney stones is increased in the early morning, when the urine is more concentrated and more acidic. Interventional measures, such as drinking a glass of water before going to sleep or alkalizing urine during the night by providing a citrate supplement, might reduce the risk of developing stones. However, these interventions have not been formally tested in prospective trials.

Glomerulonephritis. Although glomerulonephritis is a common cause of kidney diseases, little is known about how circadian rhythms affect the clinical outcome. The proteinuria that occurs in several types of nephrotic syndrome, including membranous nephropathy, follows a circadian $\operatorname{rhythm}(99,100)$; peak protein excretion occurs at around 4:00 pm and its nadir is at 3:00 am, and is independent of GFR(18). In addition, the circadian rhythm in blood pressure is markedly disrupted in patients with nephrotic syndrome, and the outcome of IgA nephropathy is dependent on the circadian rhythm of blood pressure in adults and children (101-103). Furthermore, plasma sodium levels correlate with average $24 \mathrm{~h}$ ambulatory blood pressure in patients with nephrotic syndrome (104) and thus its control might be crucial for the treatment of nephrotic syndrome.

Consistent with this idea, a combination treatment with thiazides and angiotensinconverting enzyme (ACE) inhibitors enhanced nocturnal dipping and decreased proteinuria in patients with IgA nephropathy(105).

Chronic kidney disease. Circadian rhythms in renal functions are disrupted in patients with chronic kidney disease as a result of perturbations such as nephrotic syndrome, fibrosis, hypertension and so on. Although a potential correlation between altered circadian rhythms in renal functions and progression of CKD has not been examined to date, it is clear that hypertension is one of the main markers and/or risk factors of CKD progression(106). 


\section{The circadian system in renal dysfunction}

3 Dialysis. In patients on haemodialysis or peritoneal dialysis, the circadian rhythm of several renal functions is disturbed, which might contribute to morbidity in these patients $(107,108)$. Dysregulation of melatonin secretion might partly explain the symptoms in these patients or might be, at minimum, a biomarker of the dysrhythmicity. In an uncontrolled trial, nocturnal haemodialysis improved melatonin levels and sleep efficiency and quality, which could enhance the quality of life of patients on dialysis (109).

Kidney transplantation. Renal transplantation is also associated with improvements in the quality of life for patients with end-stage renal disease (ESRD), but this effect seems to be independent of any changes in circadian rhythms or, at least, is not accompanied by substantial changes in melatonin secretion, blood pressure rhythmicity or sleep (110). Blood pressure. Several hypotheses have been proposed to explain the circadian rhythmicity of blood pressure and, in particular, the nocturnal dipping of blood pressure and its absence in hypertensive patients. A prevalent hypothesis is that the high nocturnal blood pressure in hypertensive patients maintains natriuresis and eliminates sodium that was not excreted during the day. Further support for this blood pressure-natriuresis hypothesis was provided by studies of uninephrectomy in rats and humans. Although uninephrectomy in rats did not alter the circadian rhythm of sodium, potassium and water excretion(111), uninephrectomy in humans resulted in a change in blood pressure rhythms(112), suggesting that acutely reducing the capacity of the kidney to eliminate sodium affects blood pressure rhythms.

As discussed above for fibrosis, sodium might disrupt circadian rhythms, as observed in hypertensive Dahl rats fed a high-salt diet (113). Inhibition of the sympathetic nervous system with bisoprolol in these salt-sensitive, hypertensive rats rescued the salt-induced hypertension and disruption of the sleep-wake cycle(113). However, in hypertensive patients, blood pressure levels improved after renal denervation, despite no change in sodium content in their muscle and skin, although the circadian rhythm of blood pressure was not examined in this study(114). Of note, sodium might also be involved in regulating infradian rhythms in blood pressure(1), although additional factors might also contribute to this effect. Feeding time also affects blood pressure rhythms(115). For example, the circadian rhythms of the renin-angiotensin-aldosterone system and of blood pressure 
1 were markedly shifted depending on the feeding time in dogs, suggesting that peripheral

2 clocks can be strongly entrained by nutrients and supersede other systemic cues.

3 In humans, studies of the effectiveness of treatments based on when they are 4 administered (that is, chronopharmacology) showed that blood pressure control is affected by the timing of the treatment. Thus, in a seminal study, an anti-hypertensive drug was more effective in controlling blood pressure when administrated at night than when administered in the morning (116). Several observational studies have confirmed these results; for example, taking anti-hypertensive drugs at bedtime improved blood pressure control (117), and valsartan taken at bedtime in non-dipper patients with CKD was more effective at controlling blood pressure than when taken in the morning (118). Furthermore, taking at least one anti-hypertensive drug at bedtime reduced blood pressure and the risk of cardiovascular events in hypertensive patients with chronic kidney disease (119). In the same population, CKD progression, albuminuria and glucose and lipid levels were reduced by taking one or more anti-hypertensive medications at bedtime (120).

Although the importance of chronopharmacology in the treatment of hypertension has been studied in several observational trials, only a few of these trials were randomized and controlled and most had major flaws in their design. Meta-analyses on the few existing randomized controlled trials (RCTs) confirmed that blood pressure control is improved by administering anti-hypertensive medications at bedtime in patients with hypertension and/or CKD (121-124).

The precise mechanisms by which the timing of administration improves the efficacy and/or reduces the adverse effects of a drug are not clear, but might be related to the absorption, metabolism, and/or excretion of the drug and its metabolites. Although chronopharmacology is certainly underexplored, numerous renal genes with circadian expression have been identified(31) that might be targets of known pharmacological agents, which should be validated in future studies.

Interestingly, exosomal levels of Na-Cl cotransporter (NCC; also known as SLC12A3), which is the target of thiazides, showed a circadian rhythm (that is, lower levels in the morning and higher levels in the afternoon and evening)(125). This result might partly explain why, similar to furosemide (74) discussed above, thiazides have increased efficacy when taken later during the day. 
1 In general, the sodium excretion rate seems to be the main driver of the circadian rhythmicity of blood pressure. Thus, sodium restriction can shift blood pressure from a non-dipping to a dipping pattern in patients with essential hypertension (126), which the researchers in this study suggest occurs because hypertensive patients have decreased sodium excretion during the day; therefore their blood pressure and natriuresis increase during the night to eliminate sodium and control blood pressure. Furthermore, the endothelin pathway might also have a role in the circadian rhythm of blood pressure(127, 128).

\section{Conclusions}

The multiple functions of the kidneys, which maintain the homeostasis of the whole organism, are subject to well-described circadian rhythms. The demonstration that molecular clocks exist has provided new insights into single-cell regulation. However, even as the links between renal functions and the molecular core clock are beginning to be uncovered with the demonstration of multiple levels of regulation, our overall understanding at the whole-organism level remains largely unclear. For example, the number of variables that affect blood pressure and show circadian rhythmicity, from heart function to aldosterone-sensitive tubular salt reabsorption, has complicated efforts to identify the crucial factors that control the circadian rhythm in blood pressure. Future work integrating cell-specific as well as transcriptional, translational and posttranslational rhythms will require the establishment of mathematical models that can incorporate all of this complexity(129). Studies using tissue-specific and cell-type-specific inactivation of the molecular clock will further improve our understanding of renal circadian rhythms.

Thirty four years after the initial crucial discovery of a gene involved in the molecular clock $(130,131)$, integrating the physiological and clinical relevance of circadian rhythms in the kidneys remains a challenge and should drive future research.

\section{Acknowledgments}

30 The authors thank their students, post-doctoral fellows and technicians who contributed

31 substantially to the advancement of the field through their curiosity and hard work in the 
1 laboratory. Both authors are supported by grants from the Swiss National Science

2 Foundation (31003A-169493 to D.F. and 310030-163340 to O.B.).

\section{Author contributions}

4 Both authors contributed to all aspects of the conception, content, revisions and editing

5 of this manuscript.

6 Competing interests statement

$7 \quad$ The authors declare no competing interests.

8 Publisher's note

9 Springer Nature remains neutral with regard to jurisdictional claims in published maps 10 and institutional affiliations. 
1 Table 1. The clinical relevance of circadian rhythms

\begin{tabular}{|c|c|c|}
\hline Renal condition & Putative mechanisms & References \\
\hline Fibrosis & $\begin{array}{l}\text { - Increased TGF } \beta \text { signaling } \\
\text { - Increased COX2 expression } \\
\text { - Altered endothelin pathway } \\
\text { - Altered activity of the sympathetic nervous system }\end{array}$ & $(63,87-90)$ \\
\hline Kidney stones & $\begin{array}{l}\text { - Increased excretion rate of urine salts } \\
\text { - Decreased urinary volume } \\
\text { - Reduced urinary pH }\end{array}$ & $(91-98)$ \\
\hline Glomerulonephritis & $\begin{array}{l}\text { - Membranous glomerulonephritis and nephrotic } \\
\text { syndrome: } \\
\text {-Proteinuria rhythm } \\
\text {-Control of plasma sodium levels and blood pressure } \\
\text { - IgA glomerulonephritis: } \\
\text {-Control of blood pressure }\end{array}$ & $(99-105)$ \\
\hline Blood pressure & $\begin{array}{l}\text { - Rhythm of the sodium excretion rate } \\
\text { - Reduced activity of the sympathetic nervous system } \\
\text { - Reduced endothelin pathway } \\
\text { - Change in feeding time }\end{array}$ & $(111-115,127,128)$ \\
\hline $\begin{array}{l}\text { Haemodialysis or } \\
\text { peritoneal dialysis }\end{array}$ & - Melatonin rhythm & $(108,109,132)$ \\
\hline
\end{tabular}

2 COX2, cyclooxygenase 2 ; TGF $\beta$, transforming growth factor- $\beta$.

3

4 


\section{Figure legends}

2

\section{Figure 1. Molecular clocks in humans.}

4 External cues (such as light, temperature, humidity and so on) synchronize the central

5 oscillator ('master clock') in the suprachiasmatic nucleus (SCN), which resets all

6 peripheral oscillators present in almost every cell in the body. The oscillator comprises interconnected transcription feedback loops, with BMAL1-CLOCK heterodimers on the activatory loop and PER-CRY heterodimers on the inhibitory loop. The expression of clock-controlled genes (CCGs) is driven by the output of the molecular clock and enables circadian adjustment of renal function. Retinoic acid-related orphan receptors (RORs) and REV-ERB strengthen and stabilize the feedback loops that affect BMAL1 production. ROREs, ROR response elements.

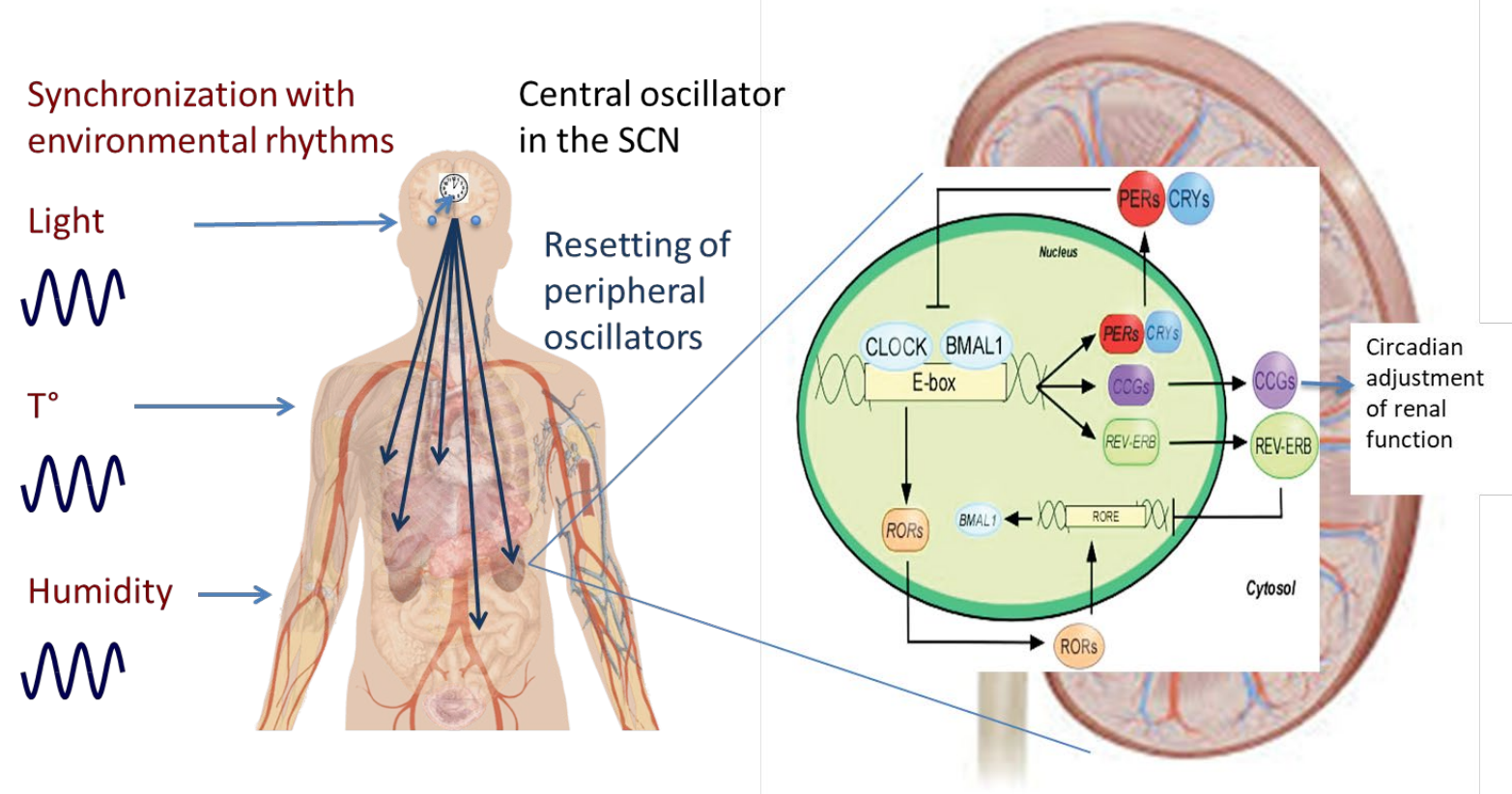

Figure 2. Intrinsic circadian clocks in the kidneys.

15 Renal plasma flow (RPF), glomerular filtration rate (GFR), podocytes and tubular cells 16 have intrinsic clocks, resulting in a circadian rhythm in urine output. Extra-renal circadian time cues (nutrients, hormones, body temperature, activity of the nervous system and so on) synchronize the rhythms of the intrinsic renal clocks. CCD, cortical collecting duct; CNT, connecting tubule; DCT, distal convoluted tubule; Glom, glomerulus; PCT, proximal convoluted tubule; PST, proximal straight tubule; TAL, thick ascending limb. 


\section{Intrinsic renal}

circadian clocks

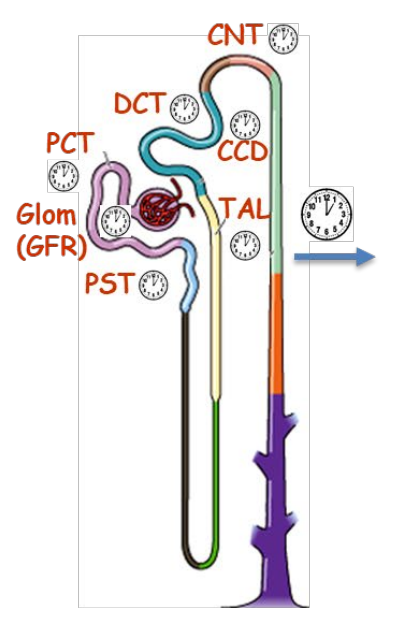

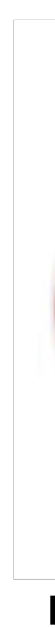

Extra-renal circadian

time cues

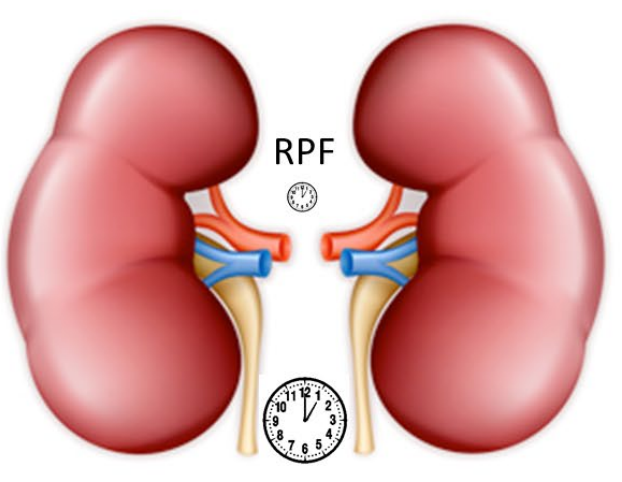

\begin{tabular}{l|l} 
Food \\
Hormones \\
Body $\mathrm{T}^{\circ}$
\end{tabular}

Neuronal activity

4 Figure 3. Intracellular circadian rhythms.

5 Numerous intracellular events display circadian oscillations, including DNA replication,

6 transcription, mRNA translation as well as protein post-translational modifications

7 (acetylation, ubiquitylation, phosphorylation and so on), targeting to the cell surface and

8 recycling and degradation. The circadian rhythms of these different processes may or may

9 not be in phase.

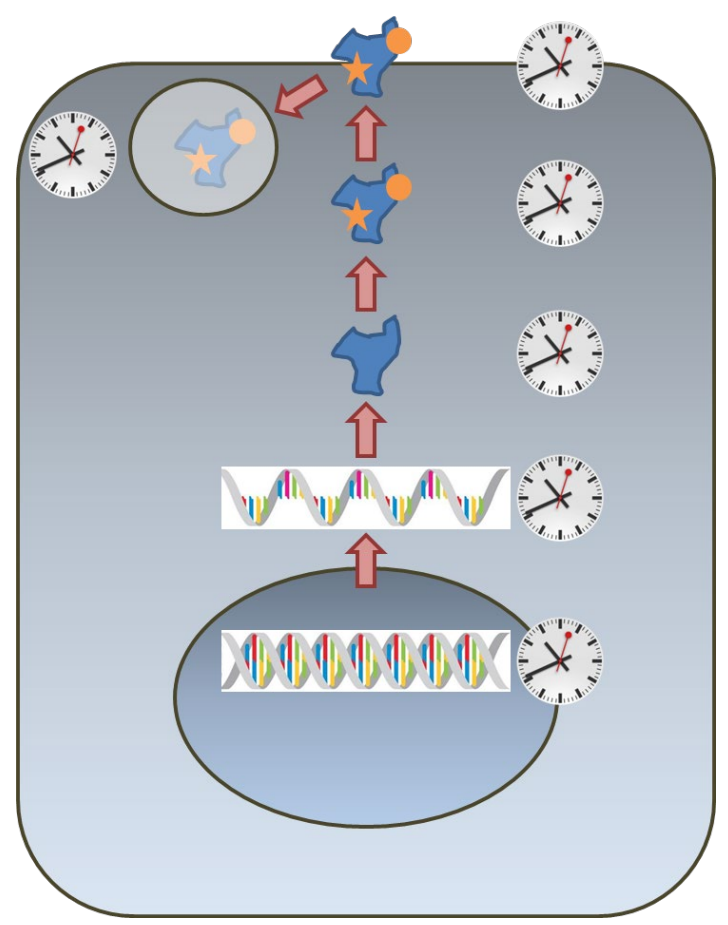




\section{References}

1. Rakova N., et al. Long-term space flight simulation reveals infradian rhythmicity in human $\mathrm{Na}(+)$ balance. Cell metabolism 17(1):125-131(2013)

2. Smith E. On the elimination of urea and urinary water, in relation to the period of the day, season, exertion, food, prison discipline, weight of body and other influences acting in the cycle of the year. Phil Trans $R$ Soc Lond 151:747834(1861)

3. Bonny 0. \& Firsov D. Circadian regulation of renal function and potential role in hypertension. Curr Opin Nephrol Hypertens 22(4):439-444(2013)

4. Firsov D. \& Bonny 0. Circadian regulation of renal function. Kidney International 78(7):640-645(2010)

5. Wuerzner G., Firsov D., \& Bonny O. Circadian glomerular function: from physiology to molecular and therapeutical aspects. Nephrol Dial Transplant 29(8):1475-1480(2014)

6. Stow L. R. \& Gumz M. L. The circadian clock in the kidney. J Am Soc Nephrol 22(4):598-604(2011)

7. Gumz M. L. Molecular basis of circadian rhythmicity in renal physiology and pathophysiology. Experimental physiology 101(8):1025-1029(2016)

8. Solocinski K. \& Gumz M. L. The Circadian Clock in the Regulation of Renal Rhythms. Journal of biological rhythms 30(6):470-486(2015)

9. Koopman M. G., et al. Circadian rhythm of glomerular filtration rate in normal individuals. Clin Sci (Lond) 77(1):105-111.(1989)

10. Tokonami N., et al. Local renal circadian clocks control fluid-electrolyte homeostasis and BP. J Am Soc Nephrol 25(7):1430-1439(2014)

11. Pons M., Forpomes O., Espagnet S., \& Cambar J. Relationship between circadian changes in renal hemodynamics and circadian changes in urinary glycosaminoglycan excretion in normal rats. Chronobiol Int 13(5):349-358(1996)

12. Nikolaeva S., et al. The circadian clock modulates renal sodium handling. J Am Soc Nephrol 23(6):1019-1026(2012)

13. Pons M., Tranchot J., L'Azou B., \& Cambar J. Circadian rhythms of renal hemodynamics in unanesthetized, unrestrained rats. Chronobiol Int 11(5):301308(1994)

14. Steele A., et al. What is responsible for the diurnal variation in potassium excretion? Am J Physiol 267(2 Pt 2):R554-560.(1994)

15. Fujii T., et al. Circadian rhythm of natriuresis is disturbed in nondipper type of essential hypertension. American journal of kidney diseases : the official journal of the National Kidney Foundation 33(1):29-35(1999)

16. Hara M., et al. Robust circadian clock oscillation and osmotic rhythms in inner medulla reflecting cortico-medullary osmotic gradient rhythm in rodent kidney. Scientific reports 7(1):7306(2017)

17. Emans T. W., Janssen B. J., Joles J. A., \& Krediet C. T. P. Circadian Rhythm in Kidney Tissue Oxygenation in the Rat. Frontiers in physiology 8:205(2017)

18. Voogel A. J., Koopman M. G., Hart A. A., van Montfrans G. A., \& Arisz L. Circadian rhythms in systemic hemodynamics and renal function in healthy subjects and patients with nephrotic syndrome. Kidney international 59(5):1873-1880(2001)

19. Somers V. K., Dyken M. E., Mark A. L., \& Abboud F. M. Sympathetic-nerve activity during sleep in normal subjects. N Engl J Med 328(5):303-307(1993)

20. Moore-Ede M. C. Physiology of the circadian timing system: predictive versus reactive homeostasis. Am J Physiol 250(5 Pt 2):R737-752.(1986) 
21. Callaway E. \& Ledford H. Medicine Nobel awarded for work on circadian clocks. Nature 550(7674):18(2017)

22. Dibner C., Schibler U., \& Albrecht U. The mammalian circadian timing system: organization and coordination of central and peripheral clocks. Annu Rev Physiol 72:517-549(2010)

23. Atger F., Mauvoisin D., Weger B., Gobet C., \& Gachon F. Regulation of Mammalian Physiology by Interconnected Circadian and Feeding Rhythms. Frontiers in endocrinology 8:42(2017)

24. Meszaros K., et al. Development of the circadian clockwork in the kidney. Kidney Int 86(5):915-922(2014)

25. Mazzoccoli G., et al. Clock gene expression in mouse kidney and testis: analysis of periodical and dynamical patterns. Journal of biological regulators and homeostatic agents 26(2):303-311(2012)

26. Wu T., et al. Regulation of circadian gene expression in the kidney by light and food cues in rats. Am J Physiol Regul Integr Comp Physiol 298(3):R635-641(2010)

27. Evans J. A. Collective timekeeping among cells of the master circadian clock. $J$ Endocrinol 230(1):R27-49(2016)

28. Fernandez D. C., Chang Y. T., Hattar S., \& Chen S. K. Architecture of retinal projections to the central circadian pacemaker. Proc Natl Acad Sci U S A 113(21):6047-6052(2016)

29. Schibler U., et al. Clock-Talk: Interactions between Central and Peripheral Circadian Oscillators in Mammals. Cold Spring Harb Symp Quant Biol 80:223232(2015)

30. Luck S. \& Westermark P. O. Circadian mRNA expression: insights from modeling and transcriptomics. Cellular and molecular life sciences : CMLS 73(3):497521(2016)

31. Zhang R., Lahens N. F., Ballance H. I., Hughes M. E., \& Hogenesch J. B. A circadian gene expression atlas in mammals: implications for biology and medicine. Proceedings of the National Academy of Sciences of the United States of America 111(45):16219-16224(2014)

32. Panda S., et al. Coordinated transcription of key pathways in the mouse by the circadian clock. Cell 109(3):307-320(2002)

33. Storch K. F., et al. Extensive and divergent circadian gene expression in liver and heart. Nature 417(6884):78-83(2002)

34. Zuber A. M., et al. Molecular clock is involved in predictive circadian adjustment of renal function. Proc Natl Acad Sci U S A 106(38):16523-16528(2009)

35. Pradervand S., Zuber Mercier A., Centeno G., Bonny O., \& Firsov D. A comprehensive analysis of gene expression profiles in distal parts of the mouse renal tubule. Pflugers Arch 460(6):925-952(2010)

36. Salhi A., Centeno G., Firsov D., \& Crambert G. Circadian expression of H,K-ATPase type 2 contributes to the stability of plasma K(+) levels. FASEB J 26(7):28592867(2012)

37. Pouly D., et al. USP2-45 Is a Circadian Clock Output Effector Regulating Calcium Absorption at the Post-Translational Level. PLoS One 11(1):e0145155(2016)

38. Jouffe C., et al. The circadian clock coordinates ribosome biogenesis. PLoS Biol 11(1):e1001455(2013)

39. Atger F., et al. Circadian and feeding rhythms differentially affect rhythmic mRNA transcription and translation in mouse liver. Proc Natl Acad Sci U S A 112(47):E6579-6588(2015) 
40. Castelo-Szekely V., Arpat A. B., Janich P., \& Gatfield D. Translational contributions to tissue specificity in rhythmic and constitutive gene expression. Genome Biol 18(1):116(2017)

41. Susa K., et al. WNK-OSR1/SPAK-NCC signal cascade has circadian rhythm dependent on aldosterone. Biochem Biophys Res Commun 427(4):743-747(2012)

42. Ivy J. R., et al. Glucocorticoids Induce Nondipping Blood Pressure by Activating the Thiazide-Sensitive Cotransporter. Hypertension 67(5):1029-1037(2016)

43. Hirano A., Fu Y. H., \& Ptacek L. J. The intricate dance of post-translational modifications in the rhythm of life. Nature structural \& molecular biology 23(12):1053-1060(2016)

44. Robles M. S., Humphrey S. J., \& Mann M. Phosphorylation Is a Central Mechanism for Circadian Control of Metabolism and Physiology. Cell metabolism 25(1):118127(2017)

45. Mauvoisin D., et al. Circadian and Feeding Rhythms Orchestrate the Diurnal Liver Acetylome. Cell reports 20(7):1729-1743(2017)

46. Rossier B. C., Baker M. E., \& Studer R. A. Epithelial sodium transport and its control by aldosterone: the story of our internal environment revisited. Physiol $\operatorname{Rev}$ 95(1):297-340(2015)

47. Hurwitz S., Cohen R. J., \& Williams G. H. Diurnal variation of aldosterone and plasma renin activity: timing relation to melatonin and cortisol and consistency after prolonged bed rest. Journal of applied physiology (Bethesda, Md. : 1985) 96(4):1406-1414(2004)

48. Doi M., et al. Salt-sensitive hypertension in circadian clock-deficient Cry-null mice involves dysregulated adrenal Hsd3b6. Nat Med 16(1):67-74(2009)

49. Adamovich Y., Ladeuix B., Golik M., Koeners M. P., \& Asher G. Rhythmic Oxygen Levels Reset Circadian Clocks through HIF1alpha. Cell metabolism 25(1):93101(2017)

50. Walton Z. E., et al. Acid Suspends the Circadian Clock in Hypoxia through Inhibition of mTOR. Cell (2018)

51. Richards J., et al. A role for the circadian clock protein Per1 in the regulation of aldosterone levels and renal Na+ retention. Am J Physiol Renal Physiol 305(12):F1697-1704(2013)

52. Solocinski K., et al. Desoxycorticosterone pivalate-salt treatment leads to nondipping hypertension in Per1 knockout mice. Acta physiologica (Oxford, England) 220(1):72-82(2017)

53. Douma L. G., et al. Renal Na Handling Defect Associated with PER1-Dependent Non-Dipping Hypertension In Male Mice. American journal of physiology. Renal physiology (2018)

54. Gumz M. L., et al. The circadian clock protein Period 1 regulates expression of the renal epithelial sodium channel in mice. J Clin Invest 119(8):2423-2434(2009)

55. Gumz M. L., et al. Regulation of alphaENaC expression by the circadian clock protein Period 1 in mpkCCD(c14) cells. Biochim Biophys Acta 1799(9):622629(2010)

56. Richards J., Jeffers L. A., All S. C., Cheng K. Y., \& Gumz M. L. Role of Per1 and the mineralocorticoid receptor in the coordinate regulation of alphaENaC in renal cortical collecting duct cells. Frontiers in physiology 4:253(2013)

57. Richards J., et al. A role for the circadian clock protein Per1 in the regulation of the $\mathrm{NaCl}$ co-transporter (NCC) and the with-no-lysine kinase (WNK) cascade in mouse distal convoluted tubule cells. J Biol Chem 289(17):11791-11806(2014) 
58. Solocinski K., et al. Transcriptional regulation of NHE3 and SGLT1 by the circadian clock protein Per1 in proximal tubule cells. Am J Physiol Renal Physiol 309(11):F933-942(2015)

59. Richards J., et al. Tissue-specific and time-dependent regulation of the endothelin axis by the circadian clock protein Per1. Life sciences 118(2):255-262(2014)

60. Ge Y., et al. Endogenously produced 20-HETE modulates myogenic and TGF response in microperfused afferent arterioles. Prostaglandins \& other lipid mediators 102-103:42-48(2013)

61. Yu M., Lopez B., Dos Santos E. A., Falck J. R., \& Roman R. J. Effects of 20-HETE on $\mathrm{Na}+$ transport and $\mathrm{Na}+-\mathrm{K}+-\mathrm{ATPase}$ activity in the thick ascending loop of Henle. Am J Physiol Regul Integr Comp Physiol 292(6):R2400-2405(2007)

62. Quigley R., Baum M., Reddy K. M., Griener J. C., \& Falck J. R. Effects of 20-HETE and 19(S)-HETE on rabbit proximal straight tubule volume transport. Am J Physiol Renal Physiol 278(6):F949-953(2000)

63. Chen W. D., et al. Circadian CLOCK Mediates Activation of Transforming Growth Factor-beta Signaling and Renal Fibrosis through Cyclooxygenase 2. The American journal of pathology 185(12):3152-3163(2015)

64. Bunger M. K., et al. Mop3 is an essential component of the master circadian pacemaker in mammals. Cell 103(7):1009-1017(2000)

65. Rudic R. D., et al. BMAL1 and CLOCK, two essential components of the circadian clock, are involved in glucose homeostasis. PLoS Biol 2(11):e377(2004)

66. Shimba S., et al. Deficient of a clock gene, brain and muscle Arnt-like protein-1 (BMAL1), induces dyslipidemia and ectopic fat formation. PLoS One 6(9):e25231(2011)

67. Kondratov R. V., Kondratova A. A., Gorbacheva V. Y., Vykhovanets O. V., \& Antoch M. P. Early aging and age-related pathologies in mice deficient in BMAL1, the core componentof the circadian clock. Genes \& development 20(14):1868-1873(2006)

68. Bunger M. K., et al. Progressive arthropathy in mice with a targeted disruption of the Mop3/Bmal-1 locus. Genesis (New York, N.Y. : 2000) 41(3):122-132(2005)

69. Boden M. J., Varcoe T. J., Voultsios A., \& Kennaway D. J. Reproductive biology of female Bmal1 null mice. Reproduction (Cambridge, England) 139(6):10771090(2010)

70. Curtis A. M., et al. Circadian variation of blood pressure and the vascular response to asynchronous stress. Proc Natl Acad Sci U S A 104(9):3450-3455(2007)

71. Xie Z., et al. Smooth-muscle BMAL1 participates in blood pressure circadian rhythm regulation. J Clin Invest 125(1):324-336(2015)

72. Sequeira Lopez M. L., Pentz E. S., Nomasa T., Smithies O., \& Gomez R. A. Renin cells are precursors for multiple cell types that switch to the renin phenotype when homeostasis is threatened. Dev Cell 6(5):719-728(2004)

73. Storch K. F., et al. Intrinsic circadian clock of the mammalian retina: importance for retinal processing of visual information. Cell 130(4):730-741(2007)

74. Nikolaeva S., et al. Nephron-Specific Deletion of Circadian Clock Gene Bmal1 Alters the Plasma and Renal Metabolome and Impairs Drug Disposition. Journal of the American Society of Nephrology : JASN 27(10):2997-3004(2016)

75. Traykova-Brauch M., et al. An efficient and versatile system for acute and chronic modulation of renal tubular function in transgenic mice. Nat Med 14(9):979984(2008)

76. Vallon V., et al. A role for the organic anion transporter OAT3 in renal creatinine secretion in mice. Am J Physiol Renal Physiol 302(10):F1293-1299(2012) 
77. Toh K. L., et al. An hPer2 phosphorylation site mutation in familial advanced sleep phase syndrome. Science 291(5506):1040-1043(2001)

78. Hirano A., et al. A Cryptochrome 2 mutation yields advanced sleep phase in humans. eLife 5(2016)

79. Xu Y., et al. Functional consequences of a CKIdelta mutation causing familial advanced sleep phase syndrome. Nature 434(7033):640-644(2005)

80. Patke A., et al. Mutation of the Human Circadian Clock Gene CRY1 in Familial Delayed Sleep Phase Disorder. Cell 169(2):203-215 e213(2017)

81. Jagannath A., Taylor L., Wakaf Z., Vasudevan S. R., \& Foster R. G. The genetics of circadian rhythms, sleep and health. Human molecular genetics 26(R2):R128R138(2017)

82. Dashti H. S., et al. Clock Genes Explain a Large Proportion of Phenotypic Variance in Systolic Blood Pressure and This Control Is Not Modified by Environmental Temperature. American journal of hypertension 29(1):132-140(2016)

83. Woon P. Y., et al. Aryl hydrocarbon receptor nuclear translocator-like (BMAL1) is associated with susceptibility to hypertension and type 2 diabetes. Proceedings of the National Academy of Sciences of the United States of America 104(36):1441214417(2007)

84. https://www.ebi.ac.uk/gwas/

85. Scheer F. A., Hilton M. F., Mantzoros C. S., \& Shea S. A. Adverse metabolic and cardiovascular consequences of circadian misalignment. Proceedings of the National Academy of Sciences of the United States of America 106(11):44534458(2009)

86. Martino T. A., et al. Circadian rhythm disorganization produces profound cardiovascular and renal disease in hamsters. American journal of physiology. Regulatory, integrative and comparative physiology 294(5):R1675-1683(2008)

87. Fletcher E. K., et al. Deoxycorticosterone/Salt-Mediated Cardiac Inflammation and Fibrosis Are Dependent on Functional CLOCK Signaling in Male Mice. Endocrinology 158(9):2906-2917(2017)

88. Speed J. S., et al. Diurnal pattern in skin $\mathrm{Na}(+)$ and water content is associated with salt-sensitive hypertension in ETB receptor deficient rats. American journal of physiology. Regulatory, integrative and comparative physiology (2017)

89. Yuan D., et al. Blue light reduces organ injury from ischemia and reperfusion. Proceedings of the National Academy of Sciences of the United States of America 113(19):5239-5244(2016)

90. Katayama T., et al. Long-term renal denervation normalizes disrupted blood pressure circadian rhythm and ameliorates cardiovascular injury in a rat model of metabolic syndrome. Journal of the American Heart Association 2(4):e000197(2013)

91. Singh R. K. \& Bansal A. Studies on circadian periodicity of serum and urinary urate in healthy Indians and renal stone formers. Progress in clinical and biological research 227B:305-313(1987)

92. Singh R. K., Bansal A., Bansal S. K., \& Rai S. P. Circadian variations of blood and urinary constituents in renal stone formers. Progress in clinical and biological research 341B:551-557(1990)

93. Singh R. K., Bansal A., Bansal S. K., Singh A. K., \& Mahdi A. A. Circadian periodicity of urinary inhibitor of calcium oxalate crystallization in healthy Indians and renal stone formers. European urology 24(3):387-392(1993) 
94. Sidhu H., et al. The loss of circadian rhythmicity of urinary solute excretion in idiopathic stone formers. British journal of urology 64(4):333-335(1989)

95. Touitou Y., et al. Alterations in circadian rhythmicity in calcium oxalate renal stone formers. International journal of chronobiology 8(3):175-192(1983)

96. Robert M., et al. Circadian variations in the risk of urinary calcium oxalate stone formation. British journal of urology 74(3):294-297(1994)

97. Bilobrov V. M., Chugaj A. V., \& Bessarabov V. I. Urine pH variation dynamics in healthy individuals and stone formers. Urologia internationalis 45(6):326331(1990)

98. Cameron M., et al. The diurnal variation in urine acidification differs between normal individuals and uric acid stone formers. Kidney international 81(11):1123-1130(2012)

99. Koopman M. G. \& Arisz L. Spectrum of diurnal rhythms in glomerular permeability in patients with membranous nephropathy. Nephrology, dialysis, transplantation : official publication of the European Dialysis and Transplant Association - European Renal Association 12 Suppl 2:47-52(1997)

100. Koopman M. G., Krediet R. T., Koomen G. C., Strackee J., \& Arisz L. Circadian rhythm of proteinuria: consequences of the use of urinary protein:creatinine ratios. Nephrology, dialysis, transplantation : official publication of the European Dialysis and Transplant Association - European Renal Association 4(1):9-14(1989)

101. Lin L., et al. Nocturnal and Circadian Rhythm of Blood Pressure Is Associated with Renal Structure Damage and Function in Patients with IgAN. Archives of medical research 47(1):25-32(2016)

102. Szelestei T., Kovacs T., Barta J., \& Nagy J. Circadian blood pressure changes and cardiac abnormalities in IgA nephropathy. American journal of nephrology 19(5):546-551(1999)

103. Skrzypczyk P., Mizerska-Wasiak M., Jerszow B., Ruszczykowski P., \& PanczykTomaszewska M. Ambulatory arterial stiffness index, blood pressure variability, and nocturnal blood pressure dip in children with IgA and Henoch-Schonlein nephropathy. Clinical nephrology 87(6):301-309(2017)

104. Haruhara K., et al. Circadian blood pressure abnormalities in patients with primary nephrotic syndrome. Clinical and experimental hypertension 39(2):155159(2017)

105. Uzu T., et al. Thiazide diuretics enhance nocturnal blood pressure fall and reduce proteinuria in immunoglobulin A nephropathy treated with angiotensin II modulators. Journal of hypertension 23(4):861-865(2005)

106. Velasquez M. T., Beddhu S., Nobakht E., Rahman M., \& Raj D. S. Ambulatory Blood Pressure in Chronic Kidney Disease: Ready for Prime Time? Kidney international reports 1(2):94-104(2016)

107. Russcher M., et al. An observational study on disturbed peripheral circadian rhythms in hemodialysis patients. Chronobiology international 32(6):848857(2015)

108. Koch B. C., Nagtegaal J. E., Hagen E. C., Wee P. M., \& Kerkhof G. A. Different melatonin rhythms and sleep-wake rhythms in patients on peritoneal dialysis, daytime hemodialysis and nocturnal hemodialysis. Sleep medicine 11(3):242246(2010)

109. Koch B. C., et al. Effects of nocturnal hemodialysis on melatonin rhythm and sleep-wake behavior: an uncontrolled trial. American journal of kidney diseases : the official journal of the National Kidney Foundation 53(4):658-664(2009) 
110. Russcher M., et al. The effects of kidney transplantation on sleep, melatonin, circadian rhythm and quality of life in kidney transplant recipients and living donors. Nephron 129(1):6-15(2015)

111. Aizman R. I., Rabinowitz L., \& Mayer-Harnisch C. Circadian rhythms and time course of adaptive sodium and potassium excretion in rats after uninephrectomy. The American journal of physiology 266(5 Pt 2):R1454-1462(1994)

112. Ohashi N., et al. The Effects of Unilateral Nephrectomy on Blood Pressure and Its Circadian Rhythm. Internal medicine 55(23):3427-3433(2016)

113. Suzuki J., et al. A critical role of sympathetic nerve regulation for the treatment of impaired daily rhythm in hypertensive Dahl rats. Hypertension research : official journal of the Japanese Society of Hypertension 33(10):1060-1065(2010)

114. Ott C., et al. Impact of renal denervation on tissue $\mathrm{Na}(+)$ content in treatmentresistant hypertension. Clinical research in cardiology : official journal of the German Cardiac Society 107(1):42-48(2018)

115. Mochel J. P., et al. Influence of feeding schedules on the chronobiology of renin activity, urinary electrolytes and blood pressure in dogs. Chronobiology international 31(5):715-730(2014)

116. Hermida R. C., Ayala D. E., Fernandez J. R., \& Calvo C. Chronotherapy improves blood pressure control and reverts the nondipper pattern in patients with resistant hypertension. Hypertension 51(1):69-76(2008)

117. Farah R., Makhoul N., Arraf Z., \& Khamisy-Farah R. Switching therapy to bedtime for uncontrolled hypertension with a nondipping pattern: a prospective randomized-controlled study. Blood pressure monitoring 18(4):227-231(2013)

118. Wang C., et al. Effect of valsartan with bedtime dosing on chronic kidney disease patients with nondipping blood pressure pattern. Journal of clinical hypertension 15(1):48-54(2013)

119. Hermida R. C., Ayala D. E., Mojon A., \& Fernandez J. R. Bedtime dosing of antihypertensive medications reduces cardiovascular risk in CKD. Journal of the American Society of Nephrology : JASN 22(12):2313-2321(2011)

120. Crespo J. J., et al. Administration-time-dependent effects of hypertension treatment on ambulatory blood pressure in patients with chronic kidney disease. Chronobiology international 30(1-2):159-175(2013)

121. Zhao P., Xu P., Wan C., \& Wang Z. Evening versus morning dosing regimen drug therapy for hypertension. The Cochrane database of systematic reviews (10):CD004184(2011)

122. Liu X., et al. Evening -versus morning- dosing drug therapy for chronic kidney disease patients with hypertension: a systematic review. Kidney \& blood pressure research 39(5):427-440(2014)

123. Wang C., et al. Chronotherapy for hypertension in patients with chronic kidney disease: a systematic review and meta-analysis in non-black patients. International urology and nephrology 49(4):651-659(2017)

124. Wang C., et al. Evening versus morning dosing regimen drug therapy for chronic kidney disease patients with hypertension in blood pressure patterns: a systematic review and meta-analysis. Internal medicine journal 47(8):900906(2017)

125. Castagna A., et al. Circadian exosomal expression of renal thiazide-sensitive $\mathrm{NaCl}$ cotransporter (NCC) and prostasin in healthy individuals. Proteomics. Clinical applications 9(5-6):623-629(2015) 
126. Uzu T., et al. Sodium restriction shifts circadian rhythm of blood pressure from nondipper to dipper in essential hypertension. Circulation 96(6):18591862(1997)

127. Dhaun N., et al. Diurnal variation in blood pressure and arterial stiffness in chronic kidney disease: the role of endothelin-1. Hypertension 64(2):296$304(2014)$

128. Johnston J. G., Speed J. S., Jin C., \& Pollock D. M. Loss of endothelin B receptor function impairs sodium excretion in a time- and sex-dependent manner. American journal of physiology. Renal physiology 311(5):F991-F998(2016)

129. Thurley K., et al. Principles for circadian orchestration of metabolic pathways. Proceedings of the National Academy of Sciences of the United States of America 114(7):1572-1577(2017)

130. Reddy P., et al. Molecular analysis of the period locus in Drosophila melanogaster and identification of a transcript involved in biological rhythms. Cell 38(3):701710(1984)

131. Bargiello T. A. \& Young M. W. Molecular genetics of a biological clock in Drosophila. Proceedings of the National Academy of Sciences of the United States of America 81(7):2142-2146(1984)

132. Koch B. C., et al. The effects of melatonin on sleep-wake rhythm of daytime haemodialysis patients: a randomized, placebo-controlled, cross-over study (EMSCAP study). British journal of clinical pharmacology 67(1):68-75(2009) 